production. Th17 cells from RA patients with high serum survivin were enriched in genes of the exhaustion set. CD4 ${ }^{+}$cells with high survivin expression were negative for PD-1, while PD-1 ${ }^{\text {hi }}$ cells had low expression of survivin. In CIA mice the survivin hiPD $-1^{-} \mathrm{CD} 4^{+}$cells were reduced by nicotine treatment $(\mathrm{p}=0.03)$ or survivin vaccination $(p=0.009)$.

Conclusions: Smoking associates with exhaustion of $\mathrm{CD} 4^{+} \mathrm{T}$ cells in RA by increasing the frequency of $\mathrm{PD}-1^{+} \mathrm{CD} 4^{+}$cells and supporting the IFN signature. Balancing T cell exhaustion and preventing the IFN signature are potential future treatment strategies for RA.

References:

[1] Frenz T, et al. J Allergy Clin Immunol 2016. 138(2): 586-589.

[2] Wasén C, et al. J Autoimmun 2017. DOI: 10.1016/j.jaut.2016.12.00.

[3] Crawford A, et al. Immunity 2014. 40(2): 289-302.

[4] Lübbers J, et al. Ann Rheum Dis 2013. 72(5): 776-780.

Disclosure of Interest: None declared

DOI: 10.1136/annrheumdis-2017-eular.6546

\section{SAT0034 ANALYSIS OF THE T-CELL SUBSET COMPOSITION IN ANKYLOSING SPONDYLITIS PATIENTS WITH LONG-STANDING ANTI-TNF THERAPY}

S. Dulic ${ }^{1}$, Z. Vásárhelyi ${ }^{2}$, A. Bajnok ${ }^{3}$, B. Szalay ${ }^{4}$, G. Toldi $^{2}$, L. Kovács ${ }^{1}$, A. Balog ${ }^{1} .{ }^{1}$ University of Szeged Departement of Rheumatology, Szeged; ${ }^{2}$ First Department of Obstetrics and Gynecology; ${ }^{3}$ First Department of Pediatrics; ${ }^{4}$ Department of Laboratory Medicine, Semme/weis University, Budapest, Hungary

Background: Ankylosing spondylitis (AS) is a chronic, progressive, immunemediated inflammatory disease, driven primarily by Th1 and Th17 cells. Anti-TNF therapies are successfully used in AS to achieve and maintain remission. However, their influence on the composition of the T-cell repertoire is not clear, and, in particular, the few published studies involve mostly patients with anti-TNF treatment of short duration.

Objectives: We aimed to characterize the changes in several T cell subsets after long-term anti-TNF treatment in AS patients.

Methods: Twenty-two AS patients on long-term anti-TNF therapy were evaluated (15 anti-TNF-responders and 7 non-responders). A wide range of cell subtypes were analysed with flow cytometry and compared with therapy-naïve and short-term data.

Results: Key findings include decreased proportions of naive CD4 and CD8 cells, increased frequencies of Th1 and Th17 cells and higher Th1/Th2 and Th17/Treg ratios in the long-term anti-TNF-treated patients (responders, non-responders and total), which was found to be significant not only when compared with healthy controls, but also with therapy-naive and short-term anti-TNF-treated AS patients. We have found several alterations within the various activated T-cell subsets increase in CD4HLADR cells in responders, in CD8HLADR cells in the whole AS group and in responders, and in CD4CD25 cells in responders, and decrease in CD4CD69 cell percentages in long-term treated patients - becoming evident only after long-term anti-TNF-therapy.

Conclusions: This study provides a comprehensive assessment of the impact of anti-TNF therapy on the T cell repertoire in AS, and indicate that these therapies induce profound changes within T-cells. Changes in T cell phenotype seem to develop progressively during therapy, even in inactive disease, and reflect an ongoing effector T-cell differentiation and activation, and a normalization of Treg development.

Disclosure of Interest: None declared

DOI: 10.1136/annrheumdis-2017-eular.6335

\section{SAT0035 INHIBITION OF PROTEIN KINASE C THETA BY THE} SELECTIVE INHIBITOR CC-90005 INDUCES T CELL ANERGY

E.M. Hur, L. Capone, J. Kosek, P.H. Schafer, G.E. Ringheim. Translational Development, Celgene Corp., Summit, United States

Background: Protein Kinase C theta (PKC- $\theta$ ), a member of the PKC family of serine/threonine kinases, is essential in T cell receptor (TCR) signaling and T cell activation [1]. Inhibition of PKC- $\theta$ activity may provide new therapeutic options for autoimmune diseases with a T effector cell dependent pathology. CC-90005 is a highly selective small molecule inhibitor of PKC- $\theta$.

Objectives: Evaluate the impact of specific PKC- $\theta$ inhibition on T-cell activation and anergy using the highly selective inhibitor CC-90005.

Methods: Human peripheral blood mononuclear cell (PBMC) or isolated T cell cultures were pre-treated with CC-90005 $(0.1-10 \mu \mathrm{M})$ and stimulated for various times up to $24 \mathrm{~h}$ with anti-CD3 and anti-CD28 activation of the TCR, followed by a washout incubation period $(1 \mathrm{~h}-24 \mathrm{~h})$. Cells were then re-stimulated in the presence or absence of CC-90005 and cultured a further 24-96 h. T cell activation was assessed by CD25 and CD69 cell surface expression, thymidine incorporation, and cytokine production.

Results: Upon TCR stimulation, CC-90005 significantly inhibited CD25 and CD69 expression and T cell proliferation in PBMC and isolated T cells. Similarly, CC-90005 inhibited the production of Th1, Th2, Th17 and other pro-inflammatory cytokines. Furthermore, the inhibitory effects of CC-90005 on T cell activation persisted after washout in stimulated T cells, indicating an induction of an anergic cell state. CC-90005 anergy induction was shown to require only short initial exposures of as little as $2 \mathrm{~h}$ and to maintain anergy for as long as 48-96 h post CC-90005 washout. However, T cells pre-treated with the inhibitor in the absence of concomitant TCR stimulation were able to respond to stimulus after as little as $1 \mathrm{~h}$ washout, indicating a rapid recovery of PKC- $\theta$ activity and T-cell function. CC-90005 upregulated an anergy-related E3 ubiquitin ligase, Gene related to anergy in lymphocytes (GRAIL) in T cells. Elevated expression level of GRAIL was maintained in unresponsive $T$ cells after washout, suggesting that GRAIL was a possible downstream mediator of CC-90005 effect on T cell anergy.

Conclusions: CC-90005, a specific inhibitor of PKC- $\theta$, significantly inhibits TCR-mediated T cell activation, proliferation, and cytokine production. Moreover, inhibition of these T-cell functions persists after drug withdrawal and restimulus of T-cells, but only if the primary $\mathrm{T}$ cell activation event occurrs in the presence of CC-90005. Thus, CC-90005 induces a functional unresponsiveness anergic state in T cells if present during TCR activation, which may have long-term therapeutic benefit in the treatment of T-cell mediated autoimmune and allergic inflammatory conditions.

\section{References:}

[1] Zhang EY, Kong K-F, Altman A. The yin and yang of protein kinase C-theta (PKC $\theta$ ): a novel drug target for selective immunosuppression. Elsevier Inc. 2013. doi:10.1016/B978-0-12-404717-4.00006-8.

Disclosure of Interest: None declared

DOI: 10.1136/annrheumdis-2017-eular.5082

\section{SAT0036 IL-17+CD8+ T CELLS ENRICHED IN THE SYNOVIAL FLUID OF PSA/SPA PATIENTS EXHIBIT A PRO-INFLAMMATORY CYTOKINE PHENOTYPE}

K.J.A. Steel ${ }^{1}$, E. Chan ${ }^{2}$, B.W. Kirkham ${ }^{2}$, L.S. Taams ${ }^{1} .{ }^{1}$ Centre for Inflammation Biology and Cancer Immunology (CIBCI), Division of Immunology, Infection \& Inflammatory Disease (DIIID), King's College London; ${ }^{2}$ Department of Rheumatology, Guy's \& St Thomas' NHS Trust, London, United Kingdom

Background: Seronegative spondylarthropathies (SpA) share common clinical and immunological characteristics, including a common association with $\mathrm{MHC}$ class I, suggesting a role for CD8+ T cells. In contrast, rheumatoid arthritis (RA) is associated with MHC class II. We previously showed that IL-17+CD8+ T cells are increased in the synovial fluid (SF) of patients with psoriatic arthritis (PsA) but not RA, compared to peripheral blood (PB). This study extends this analysis to other SpA types, and phenotypes the SF IL-17+CD8+ T cells to further elucidate their potential pathogenic role.

Methods: Paired PB and SF were collected from patients with RA, PsA and other SpA types (ankylosing spondylitis (AS), reactive arthritis $(\operatorname{ReA})$, enteropathic arthritis (EA) and peripheral SpA (p-SpA)) from Guy's Hospital Rheumatology clinic with informed consent. PB from healthy controls $(\mathrm{HC})$ was collected at King's College London. PBMC and SFMC were isolated and stimulated ex vivo with $\mathrm{PMA} /$ ionomycin in the presence of golgistop before analysis of surface marker and cytokine expression by flow cytometry.

Results: The percentage of IL-17A+CD8+ T (Tc17) cells was increased in the SF of PsA (median $0.9 \%, p=0.0012, n=13)$ as well as other $S p A$ patients $(0.34 \%$, $\mathrm{p}=0.0009, \mathrm{n}=14)$, but not RA patients $(0.07 \%, \mathrm{p}=0.3, \mathrm{n}=7)$ compared to matched PB (median $0.10 \%$, no significant differences between disease and control PB). The percentage of SF IL-17A+CD4+ T (Th17) cells was increased in patients with SpA $(2.14 \%, p=0.004, n=14), R A(2.31 \%, p=0.016, n=7)$ and to a lesser extent PsA $(0.97 \%, p=0.057, n=13)$, compared to PB (median $0.61 \%$, no significant differences between disease and control $\mathrm{PB}$ ).

Phenotypically, most SF IL-17A+CD8+ T cells expressed CCR6 (median 88\%, $\mathrm{n}=7)$ and CD161 (75\%, $\mathrm{n}=8)$, known markers of IL-17+CD4+ $\mathrm{T}$ cells. A high percentage also expressed CD103 $(69 \%, n=9)$, which was not observed in the synovial IL-17A+CD4+ T cells $(2 \%, n=10)$. A high frequency of IL-17A+CD8+ T cells co-expressed a range of pro-inflammatory cytokines including IFN- $\gamma(72 \%$, $\mathrm{n}=12)$, GM-CSF $(58 \%, \mathrm{n}=7), \mathrm{TNF}-\alpha(48 \%, \mathrm{n}=12)$ and to a lesser extent IL-21 $(20 \%, n=6)$ and IL-22 $(5 \%, n=6)$. IL-10 was usually co-expressed at very low levels $(1 \%, n=7)$, although 2 samples showed co-expression of IL-10 (33\%, $n=2)$. CCR6, CD161, GM-CSF and IL-21 were not significantly co-expressed by either IL-17A negative CD8+ or IFNg+CD8+ (Tc1) T cells from the SF, indicating this may be a co-expression profile specific to IL-17 expressing $T$ cells.

Conclusions: These findings confirm the presence of an IL-17A+CD8+ T cell subset in the SF of PsA patients and extend this to other SpA types. The expression of the integrin CD103 by these cells indicates they may represent a tissue resident memory (Trm) population in the synovial joint. In addition to $\mathrm{IL}-17 \mathrm{~A}$, the co-expression of several pro-inflammatory cytokines combined with low expression of IL-10, suggests a pro-inflammatory role for these cells.

Acknowledgements: This work was funded in part by the King's Health Partners $R \& D$ Challenge Fund (R140808) and in part by research support from Novartis.

Disclosure of Interest: K. Steel: None declared, E. Chan: None declared, B. Kirkham Grant/research support from: Novartis, UCB, Abbvie, Bristol Myer Sqibb, Celgene, Janssen, MSD, Pfizer, Roche, L. Taams Grant/research support from: Novartis and UCB

DOI: 10.1136/annrheumdis-2017-eular.5117 